\title{
On the Soundtrack of Web Series: The Untamed
}

\author{
Zhu Yunhong ${ }^{1 *}$ \\ Zhejiang Conservatory of Music, Hangzhou, Zhejiang 310013, China \\ *zhu948664@163.com
}

\begin{abstract}
In 2019, Tencent video's exclusive online play The Untamed broke the domestic online drama record with a tortuous and moving plot, and Chinese folk music is therefore highly concerned. This article will introduce the composer Lin Hai, analyse the musical form and harmony of some of the music in The Untamed and illustrate the specific role of soundtrack. As followed is a detailed form, including the relationship between music and plots.
\end{abstract}

Keywords: Soundtrack, Musical form analysis, Web series

\section{INTRODUCTION}

With the gradual maturity and expansion of domestic films and teleplays, the audience's requirements for soundtrack are also increasing. Music can strongly affect the perception of plots. Excellent works with high-quality soundtrack are a double feast in both audio and visual. Recently, fairy theme with an ancient Chinese background, represented by The Untamed, has won a large number of loyal audience at domestic and overseas. It is adapted from the novel The Founder of Diabolism by Moxiang Tongxiu. According to the data from Tencent video app, by May 2020, its broadcast volume exceeded 8 billion, which relies on its fascinating plots and incisive soundtrack. Among them, the OST (Original Soundtrack) created by composer Lin Hai was highly praised. Lin's music and plots complemented each other, which burst out wonderful effects and gave great favor to Chinese folk music.

Today, most of the domestic films and teleplays ignore the delicate music forms in the creation and arrangement of soundtrack. As its core element, it is a way to promote national culture by using diversified instruments[1]. At present, there are lack of research projects in Chinese OST. Through this paper, the author hopes to analyse the soundtrack of The Untamed, in order to broaden the vision and ideas of soundtrack and provide some suggestions in the perspective of cultural communication.

\subsection{Introduction of Composer}

Lin Hai is famous Chinese musician, composer and performer of the new century in China. In 1988, he entered the Central Conservatory of music, majoring in piano. In 2015, he was employed as the professor and master tutor of Art Department of China Media University.

Besides classical music, he innovated and studied a variety of music types, such as jazz, NEWAGE, new folk music, etc. He skillfully used the electronic MIDI and human voice in composition and arrangement. In 2000, he began to produce OST, Daming Palace, and the theme song Pipa language, which was created for the film The letter from a strange woman (2005), has been popular all over the country. Now, he has made more than 60 soundtracks, including many excellent popular films and series. He also has plenty of achievements in his personal creation. The soundtrack Huan Qin that are often used in various variety shows, skits, other web series, are from the album Pipa Xiang (2003).

As for awards, in 2002, Lin's album Moonlight border (published in 2001) won the 13th Golden Melody Award for best pop music performance album. In 2004, he was shortlisted as the 15th Golden Melody Award for best arrangement with the song Empty city (performed by Faye Wong). In 2008, he won the excellent song award of Beijing Olympic Games.

Zheng Weiwen and Chen Jialin, directors of The Untamed from Hong Kong, cooperate for the first time in the production of web series, and also the first time for them to cooperate with Lin. Their perfect combination of music and pictures achieves a high artistic effect.

\subsection{Introducion of The Untamed}

The Untamed is launched around five families: Gusu Lan family, Yunmeng Jiang family, Lanling Jin family, Qinghe Nie family and Qishan Wen family.

In youth, the five aristocratic families ruled the world together, and Wen Ruohan, the patriarch of Wen family, tried to unify the world and collect the extremely evil weapon - Yin iron left by his predecessors. Among all the young people in the family, two of the most heroic youth are Wei Wuxian (the son of Jiang's old friend) and Lan Wangji (the second son of Gusu's Lan family). They discovered the secret of Yin iron and inherited the legacy of their predecessors to protect the world. However, the Wen's family were vicious and occupied the territory of other aristocratic families. The families were overwhelmed and decided to fight back together to launch the sun shooting campaign. Meanwhile, Wei fell into the path of mass burial to cultivate the weapon called "Yin Hufu". After the 
collapse of the Wen family, Wei was set up by the traitors to protect the innocent people of the Wen family against all the families. One year later, Wei was framed by Jin Guangyao, eventually, he fell the cliff, Lan failed to recover the situation.

16 years later, Wei reappeared in Mojiazhuang as Mo Xuanyu. Lan recognized his real identity and worked with Wei fearlessly to solve the mystery under the guidance of mysterious people. With the step-by-step search, the truth was revealed to the world, and Jin died. In the end, Lan and Wei still stick to the promise and continue to move forward.

\subsection{Music Analysis}

\subsubsection{Score Analysis}

OST consists of scores and songs. Scores refers to the music part of non-songs in the film and TV, which is mainly played by instruments or hummed by people without lyrics[5]. Scores in the form of pure music gives the audience more imagination space. The soundtrack is divided into theme music, scene music and background music, having their own functions.

\subsubsection{Theme Music Unrestrained}

Theme music has a specific performance function that can bring people to a specific historical situation and cultural atmosphere or a certain emotion[2]. It is not bound by the meaning of words and can assist in many different types of description. It often uses a variety of transformations, variations, extensions, tightening and other techniques, using the melody and its tonal materials to form melody, in conjunction with the development and progression of plots, acting on the dramatic conflicts in the play.

Unrestrained highlights the main theme of two protagonists, Wei and Lan, to keep their vows and fight against negative characters. It not only expresses the ups and downs of the two protagonists' experiences, but also carries the protagonist's character of overcome difficulties and obstacles.

The piano version has the most uninhibited occurrences. Lin has released the original staff.
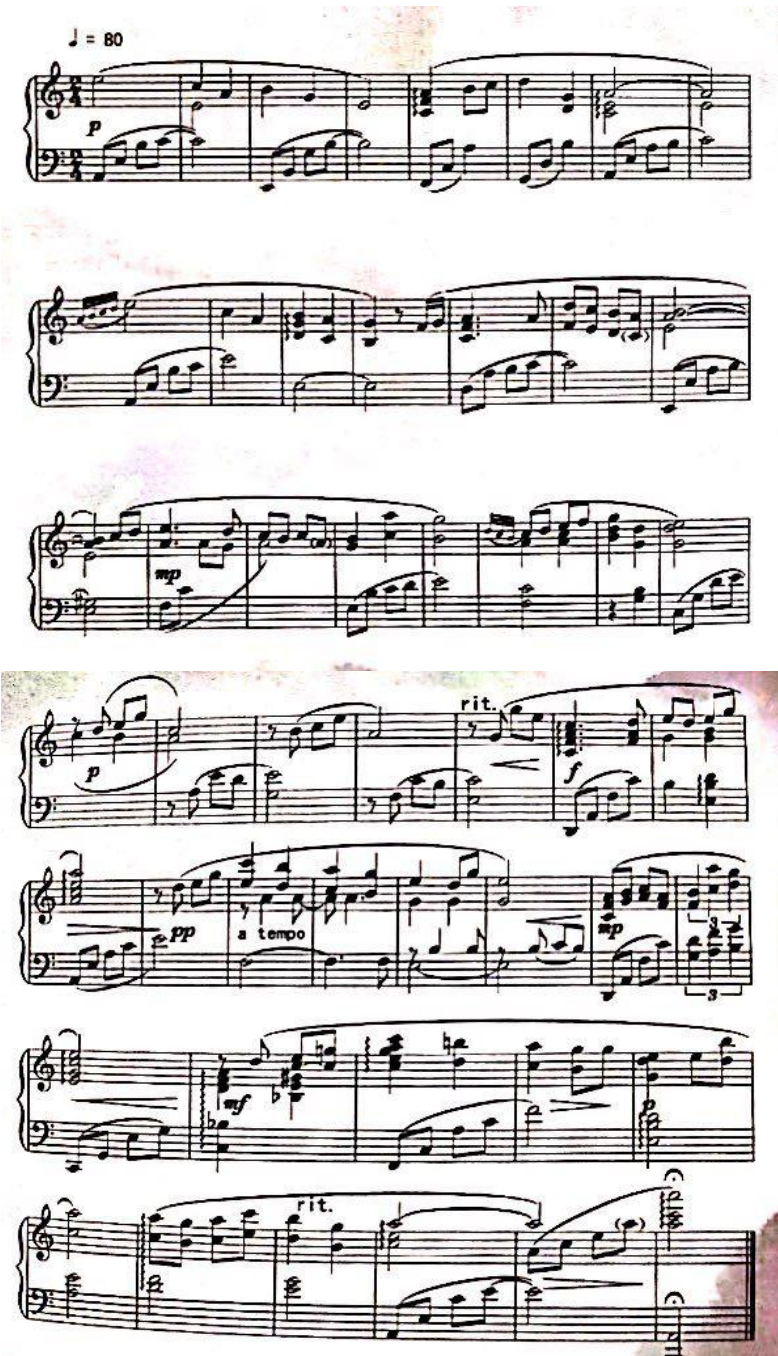

Figure 1 Staff (Version, piano): Unrestrained

The following is the analysis of harmony and melody.

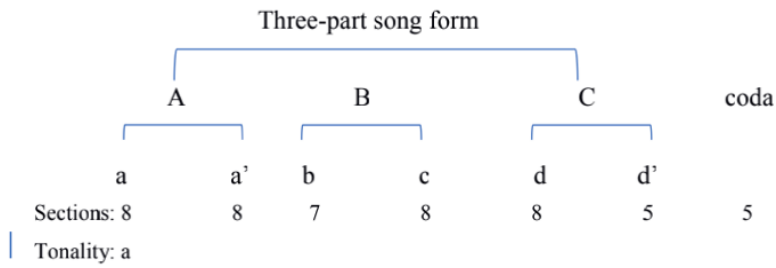

Figure 2 Musical form: Unrestrained

This song is three-part song form without recapitulation. It consists of three parts: A, B and C, and three parts use different musical materials. Therefore, it is a parallel period in a minor, with meter $2 / 4$.

Part A consists of two equal length 8 sections phrases. The a begins with descending third successively and then in a wavy motion. With a tone of narration, creating an atmosphere of lyric. The a uses the accompaniment of arpeggio, echoing part-writing, the part of harmonic progression is T-D-TSVI-DVII-T and Section VII with the 
tonic sixth chord, a perfect cadence, a diatonic transition to the a', a' uses the same musical material as a, so it is a parallel construction. Section XVI first uses dominant chord ending and preparation with dominant chord, an open phrase structure.

Part $\mathrm{B}$ begins to use different music materials, which is compared with A parts, B is a comparison period with A, the writing technique of polyphony is used in c, and the alto melody is added to the main melody, which makes it more plentiful. The part of harmonic progression is TSVI-DTSVI-DVII and ends on the third chord of the second tone, an imperfect cadence. c' uses the alternative progression of melody and accompaniment part, and the use of register expands. c' is ending on the main chord, a closed phrase structure.

Part $\mathrm{C}$ consist of two phrases of unequal length. The melody uses the same musical material, so it is a parallel construction. The $d$ begins with disjunct motion of third and fourth, pushing the song to the climax. Also uses the polyphonic music writing technique that the alto melody is added to the main melody, and the part of harmonic progression is TS-VI-D-DVII-DTIII, an imperfect cadence. The code part is added to 5 sections, the harmonic progression is K46-D-T, a perfect cadence, a closed phrase structure.

Unrestrained is the most flexible one in the use of deformation variation. It can represent the character characteristics, emotional performance and fate development of the characters in the play.

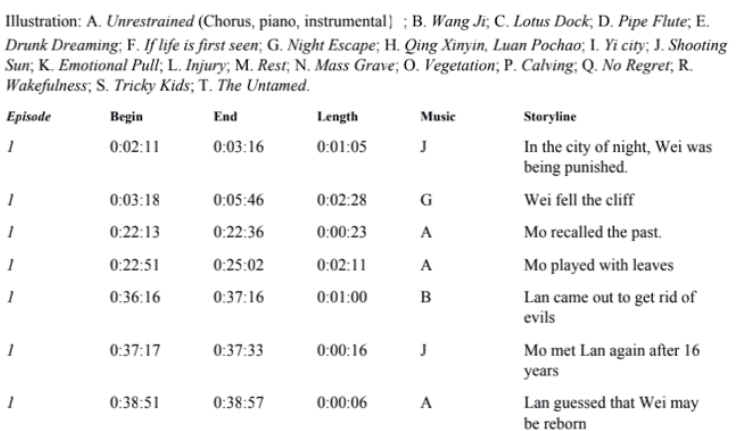

Figure $3 \mathrm{~A}$ list of the relationship between the album and the plot, episode 1

The first time it shows is in the first episode, from 0:22:13 to $0: 22: 36$. After Wei's rebirth, he saw Lan's students, so he recorded his confidant Lan in a trance. By first using the close-up shooting technique, it expanded Wei's facial emotional fluctuation[3]. Then, it used the close-up concentration to enlarge the patterns on Lan's children's clothes. Melody helps to setting off a specific emotion.

The melody of Unrestrained is mature in the use of deformation, variation, extension, contraction and so on. It uses different accessories such as piano, cello, bamboo flute and so on to impact diversely.

\begin{tabular}{|c|c|c|c|c|c|}
\hline Episode & Begin & End & Length & Music & Storyline \\
\hline 3 & $0: 09: 32$ & 0:11:05 & 0:01:33 & $\mathrm{F}$ & $\begin{array}{l}\text { Jiang Yanli first met Jin } \\
\text { Zixuan }\end{array}$ \\
\hline 3 & $0: 13: 46$ & $0: 14: 13$ & $0: 00: 27$ & B & Lan first met Wei \\
\hline 3 & $0: 21: 23$ & 0:23:03 & 0:01:40 & A & $\begin{array}{l}\text { Wei violated rules and } \\
\text { fought with Lan }\end{array}$ \\
\hline 3 & $0: 23: 35$ & $0: 25: 15$ & $0: 01: 40$ & A & $\begin{array}{l}\text { Lan took Wei to see the } \\
\text { rules }\end{array}$ \\
\hline 3 & $0: 29: 38$ & 0:30:03 & $0: 00: 25$ & A & Wei apologized to Lan \\
\hline 3 & $0: 32: 26$ & 0:34:00 & $0: 01: 34$ & I & $\begin{array}{l}\text { Many people searched for } \\
\text { evil spirits }\end{array}$ \\
\hline 3 & $0: 36: 41$ & $0: 38: 14$ & 0:01:23 & $\mathrm{N}$ & $\begin{array}{l}\text { Wen Ruohan assigned Xue } \\
\text { Yang to look for other } \\
\text { pieces of Yin iron }\end{array}$ \\
\hline
\end{tabular}

Figure 4 Episode 3

In episode 3, from 0:21:23 to 0:23:03, Lan fought with Wei for the first time. The segment uses the slow melody of its chorus version and the orchestral performance, plus the collision sound of their psychological activities and swords, and the coordination of soundtrack and onomatopoeia[4]. The musical and pictorial alignment makes the initial discord in the plots of the two people form a strong contrast with the melodious and broad music.

$\begin{array}{llllll}\text { Episode } & \text { Begin } & \text { End } & \text { Length } & \text { Music } & \begin{array}{l}\text { Storyline } \\ 36\end{array} \\ 36: 02: 46 & 0: 03: 59 & 0: 01: 13 & \mathrm{~A} & \text { Lan drunk and lethargic } \\ 36 & 0: 04: 04 & 0: 05: 46 & 0: 01: 42 & \mathrm{~A} & \begin{array}{l}\text { Wei piped to summon Wen } \\ \text { Ning }\end{array} \\ 36 & 0: 08: 38 & 0: 09: 57 & 0: 01: 19 & \mathrm{R} & \text { Wei directed Wen } \\ 36 & 0: 15: 40 & 0: 18: 00 & 0: 02: 20 & \mathrm{~A} & \text { Wei teased Lan } \\ 36 & 0: 18: 21 & 0: 19: 50 & 0: 01: 29 & \mathrm{~A} & \begin{array}{l}\text { Lan revealed his true } \\ \text { feelings after drinking }\end{array}\end{array}$

Figure 5 Episode 36

In episode 36 , from 0:18:21 to 0:19:50, Lan regretted that he did not advance and retreat with Wei sixteen years ago, but Wei hoped he would not blame himself. Here uses close range and close-up shooting, the melody playing with cello and piano, setting off the dignified atmosphere of them.

\subsubsection{Scene Music Qing Xinyin, Luan Pochao}

Scene music refers to music used in a single scene that only plays a role in specific scenes. Scene music is mainly aimed at the plots in a specific scene, as well as the foreshadowing, rendering, and foil the ups and downs of the psychological activities of the characters in this scene. It can often help the emergence of some dramatic climaxes and the continuous development of the whole plots, creating artistic conception, etc. It is usually set for a certain scene, which is basically non-repetitive.

Qing Xinyue, Luan Pochao is one of the typical scene music, and it is also a representative active music. When it appears, there are clear musical instrument performance pictures in the play.

The following is the analysis of harmony and melody. 


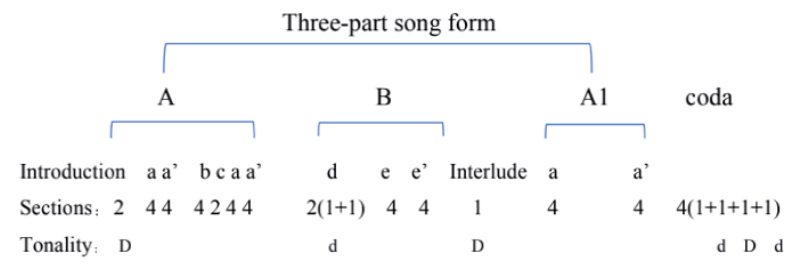

Figure 6 Musical form: Qing Xinyue, Luan Pochao

This song is three-part song form with recapitulation. It consists of three parts: A, B, and A1. A1 uses the same musical materials as A. It has 5 modulations totally, with meter $4 / 4$ and $6 / 4$.

Part A: $4+4+4+2+4+4$ six phrase paragraphs, it is a contrasting double period. The part of a a' b' consists of three equal length 4 sections phrases. The a and a' use dotted notes and 16th-notes frequently, which highlight the strength of the melody. One section of c changes the meter to $6 / 4$ and prepares for repetition. In the last phrase, $\mathrm{c}$ is ending on the dominant, there is a feeling of unfinished narration, immediately following the next period.

Part B: $2+4+4$ three phrase paragraphs, $d$ begins to modulation that turn major to minor, natural transition to next part. The rhythm of e is suddenly compact, add 32ndnotes, creating a completely different sound atmosphere. The use of several minor second and minor third makes the minor tonality more obvious, the whole melody lingers in a register, the final fall of the main tone to e'. This part rhythm is similar with e and bold to add higher tone, for melody up and compact.

Part A1: 4+4 two phrase paragraphs, using the same material of a a', without any changes, through the interlude end on the dominant, immediately turn back to the major and start from the tonic. The code uses the same musical note, and has modulations completed 3 times.

$\begin{array}{llllll}\begin{array}{l}\text { Episode } \\ 41\end{array} & \begin{array}{l}\text { Begin } \\ 0: 19: 20\end{array} & \begin{array}{l}\text { End } \\ 0: 20: 20\end{array} & \begin{array}{l}\text { Length } \\ 0: 01: 00\end{array} & \begin{array}{l}\text { Music } \\ \mathrm{H}\end{array} & \begin{array}{l}\text { Storyline } \\ \text { Lan Xichen and Jin } \\ \text { Guangyao played Qing } \\ \text { Xinyin for Nie }\end{array} \\ 41 & 0: 21: 10 & 0: 22: 27 & 0: 01: 17 & \mathrm{H} & \begin{array}{l}\text { Jin played Luan Pochao } \\ \text { with selfishness }\end{array} \\ 41 & 0: 22: 28 & 0: 23: 02 & 0: 00: 34 & \mathrm{I} & \begin{array}{l}\text { Nie heard the sound, then } \\ \text { felt be perplexed in mind }\end{array} \\ 41 & 0: 23: 08 & 0: 23: 29 & 0: 00: 21 & \mathrm{H} & \begin{array}{l}\text { Lan played Qing Xinyin to } \\ \text { wake Wei }\end{array} \\ 41 & 0: 24: 11 & 0: 24: 24 & 0: 00: 13 & \mathrm{I} & \begin{array}{l}\text { Nie attacked Jin } \\ \end{array} \\ & & & & \end{array}$

Figure 7 Episode 41

Both Qing Xinyin and Luan Pochao represent the unique music of the Lan family in the plots, but there are good and evil points. The song appeared 12 times in total. In episode 41, from 0:21:10 to 0:22:27, Jin copied the disordered soul into the Qing without permission, which made Nie's mood disordered. During the shooting, he gave a close-up of Guqin, the active music made the song not inconsistent with the scene. From Qing Xinyin to Luan Pochao, it naturally tunes the tune and uses the simultaneous description technique of music and painting, the facial expressions of the characters are shot close-up, so that the song plays a role of rendering outside the plots and promotes the climax of the story in the plots.

\subsubsection{Background Music The Untamed}

Background music is a kind of special scene music, which is often used to identify the environment, occasion, region, etc., it can be used to emphasize the authenticity of the scene in the plots, at the same time, it also can depict the psychological activities of the characters.

The following is the analysis of harmony and melody.

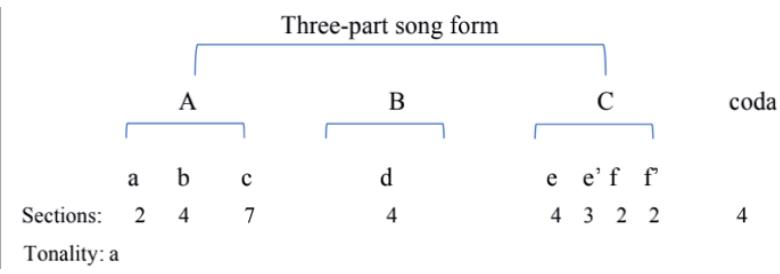

Figure 8 Musical form: The Untamed

This song is three-part song form without recapitulation. It consists of three parts: A, B and C, and three parts use different musical materials. It is a parallel period in a minor, with meter $4 / 4$.

Part A: $4+4+7$ three phrase paragraphs. The first four sentences have the same rhythm and have same tone except for the second and fourth section, the pitch is octave and end on the dominant, beginning to rise mood. The part of harmonic progression is I-V. The figure is dominated by 8th-notes. There are some ties, making the rhythm of the strength more dynamic. The first two phrases are square and parallel, at the beginning of the part, the melody ascending, the third phrase descending is reversed to form a contrast.

Part B: One phrase, with one-stop characteristics. Around the development of the main tone, using 16th-notes and 8thnotes and change the speed, from the first part, and add \#a to make minor second for more dramatic. The last two sections are using consecutive fifths intervals for parallel motion, which makes the voice full of tension, naturally connecting with the exciting third part later.

Part C: It is composed of 4 unequal length phrases. This melody is mostly using dotted note, which makes the strength become richer. Both e and e' go ascending and then descending, the fluctuation is obvious, then both $\mathrm{f}$ and $\mathrm{f}^{\prime}$ are going descending, so the overall melody line shows a descending trend. The coda ends with a long main vocal of two sections, consolidating the overall tonality.

$\begin{array}{lllll}\text { Episode } & \text { Begin } & \text { End } & \text { Length } & \text { Music } \\ 33 & 0: 05: 28 & 0: 05: 41 & 0: 00: 13 & \mathrm{~A} \\ 33 & 0: 09: 34 & 0: 13: 15 & 0: 03: 41 & \mathrm{G} \\ 33 & 0: 13: 20 & 0: 17: 57 & 0: 04: 37 & \mathrm{~A} \\ 33 & 0: 18: 05 & 0: 20: 33 & 0: 02: 28 & \mathrm{~F} \\ 33 & 0: 22: 05 & 0: 23: 47 & 0: 01: 42 & \mathrm{~A} \\ 33 & 0: 25: 33 & 0: 25: 47 & 0: 00: 14 & \mathrm{I} \\ 33 & 0: 27: 27 & 0: 30: 04 & 0: 02: 31 & \mathrm{M} \\ 33 & 0: 28: 06 & 0: 28: 56 & 0: 00: 50 & \mathrm{~A} \\ 33 & 0: 39: 47 & 0: 40: 43 & 0: 00: 56 & \mathrm{~T}\end{array}$

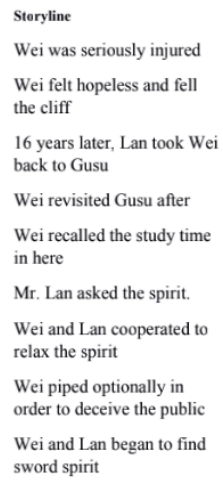

Figure 9 Episode 33 
It appeared four times in the play totally. In episode 33, from 0:39:47 to 0:40:43, after Wei was reborn, he decided to go down the mountain with Lan to find the truth of that year. Through the shot of passive music appearing together at this time to shoot in the perspective, with the montage technique of time-space music. The tone of the whole song of The Untamed rises slowly from the beginning to the last grand momentum, which symbolizes the ups and downs of the protagonist's fate and the trend of the plots.

\subsection{Analysis Lyrics of Theme songs}

This paper presents some improvements based on the The charm of the theme song is reflected in the melody and the lyrics. It can enrich the main line of the play, express some feelings and set off specific scene atmosphere with specific style and concise emotion.

Unrestrained is the episodic theme song. It plays a highly generalized role in the plots, also reveals the ups and downs of the two protagonists' experiences. Among them, its lyrics set up an image for the song, endow its soul, and more clearly foretell the fate and emotion of the main characters.

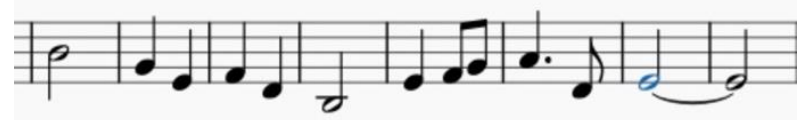

Accord with the flute, I gazed at the cloud hidden in the ever-ended night, lonely and blue

Figure 10 Lyrics in Unrestrained

These two lines are for Wei and Lan. In youth, they experienced a lot of resentment. Wei was puzzled and opposed by the world because he created his own tricks. The melody of the two sentences is exactly the same, which means a dialogue between the protagonists.

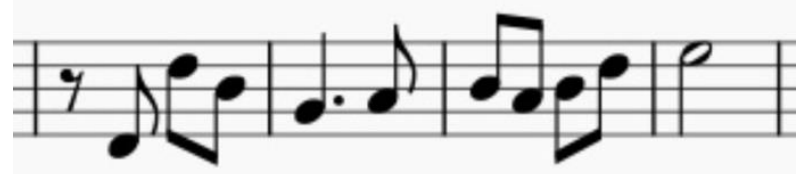

Reed flowers grows under the frost-like moonlight, always telling the lingering love.

\section{Figure 11 Lyrics in Unrestrained}

The reed flower, a metaphor for Wei's and Lan 's life path. They are always fighting alone as same as reed flower swaying in the wind.

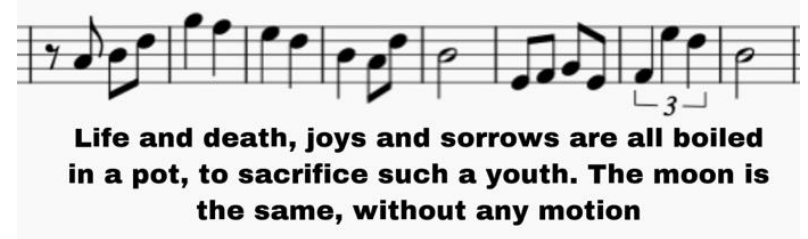

Figure 12 Lyrics in Unrestrained
Lan and Wei experienced the tribulation, which make them unable to be carefree like a youth. The first two lyrics, the melody goes up and down through the three sounds after the ascending, and echoes the lyrics, which is a sinking emotion. In the penultimate section, the tune goes a disjunct motion, it strength the mood of interrogative words.

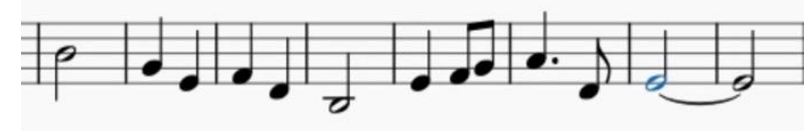

Walking through mountains and rivers, you cannot finish your journey but will meet vast crowd.

Figure 13 Lyrics in Unrestrained

When Wei disappeared, Lan looked for Wei everywhere, walked through many places, just to find him. These lyrics were sung by Lan alone, here the timeline in the lyrics has been 16 years later, the melody reproduces the first paragraph and adds a new drumbeat, with the meaning of going forward again.

Unrestrained arranges the choral version. There are two protagonists singing the lyrics separately, which is full of the characters' journey in the play. This part of the lyrics to echoes the plots, in the solo part is the question and answer of two protagonists; in the chorus parts is the summary, bursting out a stronger auditory effect and feeling.

\subsection{Social Impact}

The Untamed has set off unexpected repercussions in 2019, it is attribute to the excellent plots, actor's acting skills, costume props, dubbing team, the OST, etc., so it has brought a special fan effects in diverse aspects. For example, Ciga long, is a brand of lipstick, it inspired consumers' passion for shopping, because it asked designers to design and package new lipstick, specifically taking five families as the creative elements, combined

with their own characteristics and unique style of net series to the new lipstick.

OST has different aesthetic characteristics, and the scope of its role is expanding. The Untamed is an ancient costume series with the theme of fairy and the music full of national elements has played an extraordinary work. Some success factors are the OST both national and modern, the combination of novel orchestration techniques and traditional instruments.

Lin integrated the national musical instruments such as pipa, bamboo flute, zither into the music, and publicized the important value and confidence of national culture through the dissemination of works. He arranges the music with the plots as the main intention, which shortens the distance between the play and the audience. Moreover, it shows the unique national culture of soundtrack in China, bringing Chinese folk music to the public's vision. The popularity of The Untamed even expand to Thailand, South Korea, Japan and other countries. Many people began to learn national 
musical instruments such as bamboo flute and show their study for Chinese folk music on social media.

\section{CONCLUSION}

OST is active in the public's vision in a unique form. Music promotes the audience's empathy with plots, meanwhile it conveys rich emotional cognition. In The Untamed, each piece complements the plots and creates a perfect audiovisual picture. Lin integrates the elements of his nation into the trend of the times, pushes the Chinese folk culture to the public and embarks on a broader stage. An increasing number of people pay attention to the unique style music of our country, which is a music creation and propaganda form with special value.

\section{REFERENCES}

[1] David, B., Kristin, T. (2009) Film Art: An Introduction. McGraw-Hill, New York.

[2] Fred, K., Wright, R. (2004) On the Track: A Guide to Contemporary Film Scoring. $2^{\text {nd }}$ ed. Routledge, London, New York.

[3] Michael, R. (1989) Directing: Film Techniques and Aesthetics. Focal Press, Oxford.

[4] Tomlinson, H. (2010) Sound for Film and Television. Focal Press, Oxford.

[5] Zeng, T., Wei L. and Chen X. (2017) Film TV series Music Analysis. $2^{\text {nd }}$ ed. Communication University of China Press, Beijing. 